\title{
Critical Review on Impact of COVID 19 and Mental Health
}

\author{
Jaya Pranoykumar Gawai ${ }^{1}$, Seema Singh², Vaishali Deoraoji Taksande ${ }^{3}$, \\ Tessy Sebastian ${ }^{4}$, Pooja Kasturkar ${ }^{5}$, Ruchira Shrikant Ankar6
}

\begin{abstract}
${ }^{1}$ Department of Mental Health Nursing, Datta Meghe Institute of Medical Sciences (Deemed to be University), Smt. Radhikabai Meghe Memorial College of Nursing, Wardha, Maharashtra, India. 2Department of Medical Surgical Nursing, Datta Meghe Institute of Medical Sciences (Deemed to be University), Smt. Radhikabai Meghe Memorial College of Nursing, Wardha, Maharashtra, India. ${ }^{3}$ Department of Obstetrics and Gynaecology, Datta Meghe Institute of Medical Sciences (Deemed to be University), Smt. Radhikabai Meghe Memorial College of Nursing, Wardha, Maharashtra, India. ${ }^{4}$ Department of Mental Health Nursing, Datta Meghe Institute of Medical Sciences (Deemed to be University), Smt. Radhikabai Meghe Memorial College of Nursing, Wardha, Maharashtra, India. ${ }^{5}$ Department of Mental Health Nursing, Datta Meghe Institute of Medical Sciences (Deemed to be University), Smt. Radhikabai Meghe Memorial College of Nursing, Wardha, Maharashtra, India. ${ }^{6}$ Department of Medical Surgical Nursing, Datta Meghe Institute of Medical Sciences (Deemed to be University), Smt. Radhikabai Meghe Memorial College of Nursing, Wardha, Maharashtra, India.
\end{abstract}

\section{ABSTRACT}

\section{BACKGROUND}

The NOVEL COVID 19 pandemic's blowout from the PRC with its spread across the Globe has left a heavy impact on the mental health of the people. Measures and strategies evolved to contain the virus are public awareness, personal protection and hygiene, social distancing, testing kits etc. The related mental health issues are to be addressed. We wanted to evaluate the impact of COVID19 pandemic on the mental health of the people across the Globe.

\section{METHODS}

Mental health related to COVID 19 outbreak, for the last five months from December 30, 2019: the published articles; abstracts; editorials; copyrights, have been considered and reviewed, from Sources: PubMed; Medline; and Cochrane database library.

\section{RESULTS}

The physical and social distancing has resulted as social isolation. The self-quarantine has led many to anxiety, depression, mood changes, insecurity, low self-esteem and post-traumatic stress disorder. Many are may not be able adapt to present situation.

\section{CONCLUSIONS}

More Interventional studies are required to attend mental health during / after this Pandemic. Encouraging productive activities at home can prevent the mental disorders. Proper structuring cognizance by the institutions and online services is required to educate and rehabilitate the victims across the country and globe.

\section{KEY WORDS}

Outbreak, COVID 19, Pandemic, Impact, Mental Health, Quarantine.
Corresponding Author:

Jaya Pranoykumar Gawai,

Department of Mental Health Nursing,

Datta Meghe Institute of Medical Sciences

(Deemed to be University), Smt. Radhikabai Meghe Memorial College of Nursing,

Wardha, Maharashtra, India.

E-mail:jayapranoykumargawai@gmail.com

DOI: $10.14260 /$ jemds/2020/470

How to Cite This Article:

Gawai JP, Singh S, Taksande VD, et al. Critical review on impact of COVID 19 and mental health. J. Evolution Med. Dent. Sci. 2020;9(30):2158-2163, DOI: $10.14260 /$ jemds $/ 2020 / 470$

Submission 18-04-2020,

Peer Review 18-06-2020,

Acceptance 24-06-2020,

Published 27-07-2020.

Copyright (C) 2020 JEMDS. This is an open access article distributed under Creative Commons Attribution License [Attribution 4.0 International (CC BY 4.0)] 


\section{BACKGROUND}

The COVID -19 became a pandemic in a rapid succession. The fear gripped the entire world. Rising number of affected and the death count has reached an alarming stage. Mental health care professionals are challenged with an uphill task. Professional handling of the affected, daily deaths, self-care and quarantines take a lot of courage to face the fact in this times of crisis.(1)

Novel corona virus disease 2019 (COVID-19) is now extensive across the globe. having horror-struck more than 640,000 people and indicted over 30,000 lives in approximately 202 countries with no sign of slowing down (World Health Organization, 2020).(2)

In its initial phase of the COVID-19 outbreak, the article reported, more than half of affected with moderate-to-severe, and about one-third with moderate-to-severe anxiety.(3) In Italy, $25 \%$ of the population was attributed with neuropsychiatric disorders. However psychologically stating, the period of stress in social isolation, is seen as a risk to mental disorders: like anxiety; mood disorders; addiction; thought disorders; objective / subjective feelings of loneliness; suicidal tendencies domestic abuse/harassment etc. Due to pandemic crisis, untimely deaths of family members or loved ones, reflects as complicated grief associated with social isolation. This effect has increased further with a lack of proper support system.

COVID-19 is challenging the situation across the globe. In well-connected world regardless of geographic detachment, we are deeply concerned as this illness is a threat to our physical and mental wellbeing. According to psychiatric perspective, COVID-19 remains a challenge situation for the mental health care team members to act immediately and deal with this very public health crisis. ${ }^{(4)}$

During the recent pandemic, social isolation and selfquarantine may precipitate as depression and anxiety. The people may get detached from their loved ones, with deprived personal liberties, and empty purpose because of altered routine lifestyle and means of livelihood. This is seen as influencing people with frustration, boredom, insecurity at home at work place, mood imbalance, and untoward casualties.

Dealing with the mental health issues in view of this pandemic, requires a comprehensive mental health care team members, public administration and policy makers with promising result-oriented strategies. Enhancing healthy lifestyle, hobbies, virtual social interactions and mindfulness needs to be addressed. The mass media has an important role to play in bringing about a successful implementation of social distancing, yet curbing the adverse effects on mental health, challenges the society returning to normalcy after social distancing remain to be explored.(5)

The psychological problems the medical and paramedical staff are to be addressed on a war-footing. Especially health care workers, and general public front-line workers are under a constant threat being infected and affected on socio economic levels, during the spread while and controlling COVID-19. Early developed strategies, to prevent and treat vicarious traumatisation among the medical staff and general public are extremely necessary.(6)
As an important step to provide necessary mental health support, the mental health professional is to be involved in the direct management in this crisis situation.

\section{Resilience COVID-19}

To provide psychological support to health care professionals and laypeople. Therefore, the mental health professionals will be able to take initiatives such as to prevent future epidemics and dramatic development of psychiatric disorders.(7)

\begin{tabular}{|cc|}
\hline Sources & $\begin{array}{c}\text { Reviews Collected from PubMed, Medline, } \\
\text { and Cochrane Database Library }\end{array}$ \\
Methodology & $\begin{array}{c}\text { Quantitative } \\
\text { Evidences } \\
\text { Collected from published articles, review articles, abstracts, editorials } \\
\text { and copyright, newspaper, government's reports. Trials are needed to } \\
\text { develop effective treatment for COVID-19. More research is needed to } \\
\text { identify gaps between treatment and services. }\end{array}$ \\
& $\begin{array}{c}\text { Structured interventions to be planned for mental health issues. } \\
\text { Compulsive Screening test to be developed for pandemic Gaps to be } \\
\text { Conclusions } \\
\text { built immediately. Policy makers should insist on further research with } \\
\text { proper funding and recognition. Scoping review should be } \\
\text { recommended to identify magnitude of CoviD-19. }\end{array}$ \\
\hline Table 1. Methods
\end{tabular}

\section{PSYCHOLOGICAL IMPACT OF COVID 19}

- Anxiety.

- Depression.

- Substance abuse disorders with increased risk of infection with COVID 19.

- Post-traumatic stress disorder.

Uncertainty of this pandemic period is disrupting people's life and their relations. There is profound and wide spectrum of psychological impact that such outbreaks can inflict upon people's life (Lima et al., 2020). Many can develop mental illnesses as family care givers will have to tackle extra burden. Most health professionals working in isolation units and hospitals may need to be trained to handle mental health issues during and post COVID-19.

(Lima et al., 2020). Barbisch et al. (2015) described how the quarantine, "caused a sense of collective hysteria, leading the staff to desperate measures". Suicidal cases were reported where the infected nurses committed suicide in a span of a few days, it is suspected these cases were probably due to fear of spreading COVID-19 among patients. The possibility of developed fear and anxiety of falling sick or dying, and the feeling of helplessness will lead to an increase in the 2020 suicide rates.(8)

The article consistently reported the benefit of quarantine, as confirmed cases reduced, suspected cases averted and related death rates slowed down. Very low-certainty evidence indicated that the effect of quarantine on reducing incidence and deaths was small. When the models combined quarantine with other prevention and control measures: school closures; travel restrictions and social distancing, demonstrated a larger effect on the reduction of new cases, transmissions and deaths than individual measures alone.(9) 


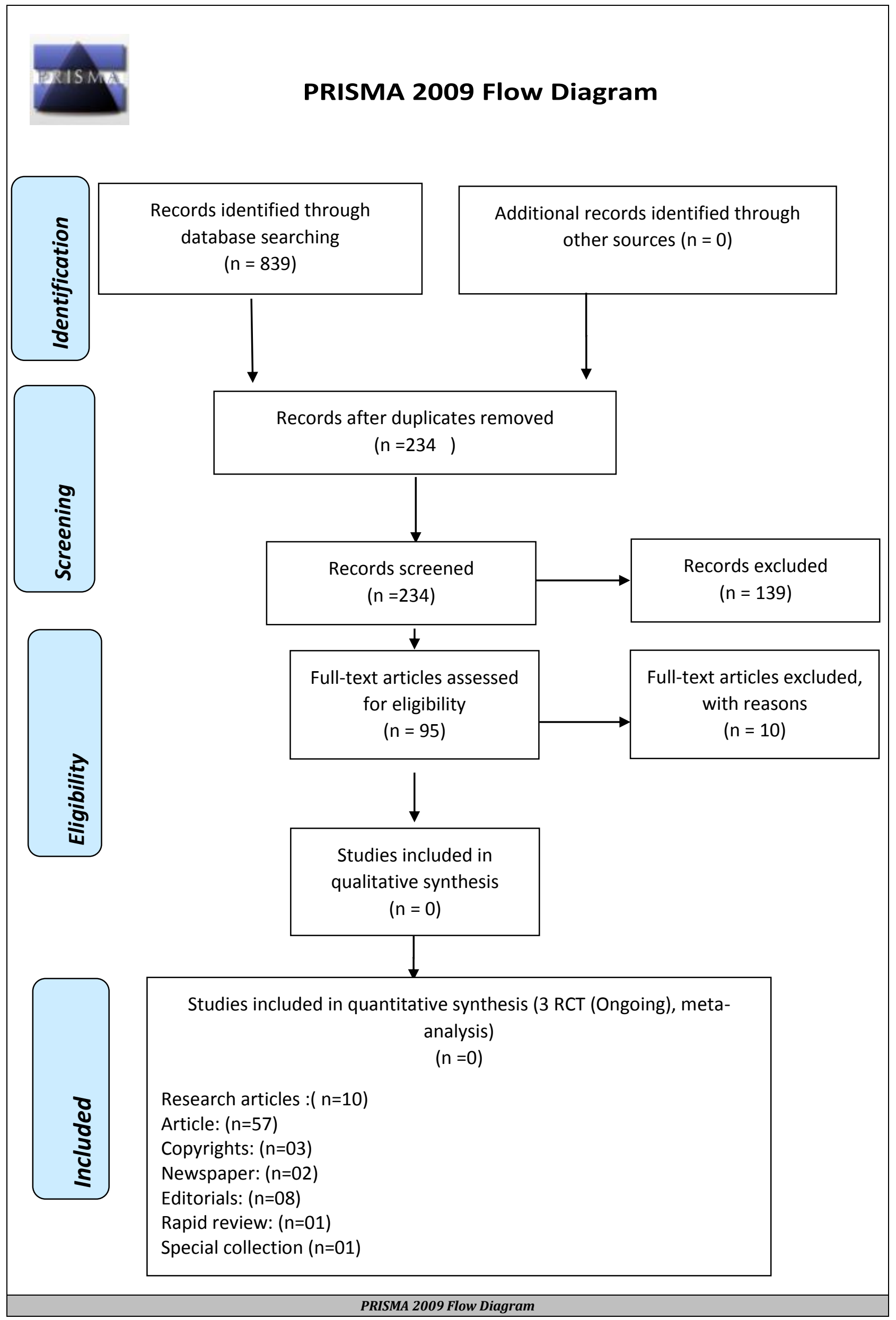




\section{Suggestions to Minimize the Psychological Effects of COVID-19 Pandemic}

- Shifting of mental health professionals from one place to other should be avoided for better outcome.

- Psychological interventions for the communities affected by Covid-19, people at high risk of psychological morbidity, should get mental health literacy through

- online and smart phone technologies as a preventive measure.

- Need to focus on frontline workers: ASHA workers, with proper sanitization knowhow to reduce secondary traumatic stress reactions, awareness of outbreaks and its symptoms, compulsive planned layoffs, self-care, media coverage and with prioritized SOS calls.

- Create awareness of risk among employers, enhanced peer-support, and practical assistance for healthcare workers.(10)

\section{Indicators of Psycho-Social Consequences}

- Vulnerability to biological and psycho-social stressors.

- Fear of exposure to infection and no treatment for COVID 19

- Lack of resources

- Unnecessary information through mass media channels.(11)

Fear seems more certainly a consequence during mass quarantine.(12) Sijia L. reported, negative emotions (anxiety, depression and indignation) and sensitivity to social risks at increased levels, while the scores of positive emotions (happiness) and life satisfaction are on a decreasing trend during the pandemic of COVID-19.(13)

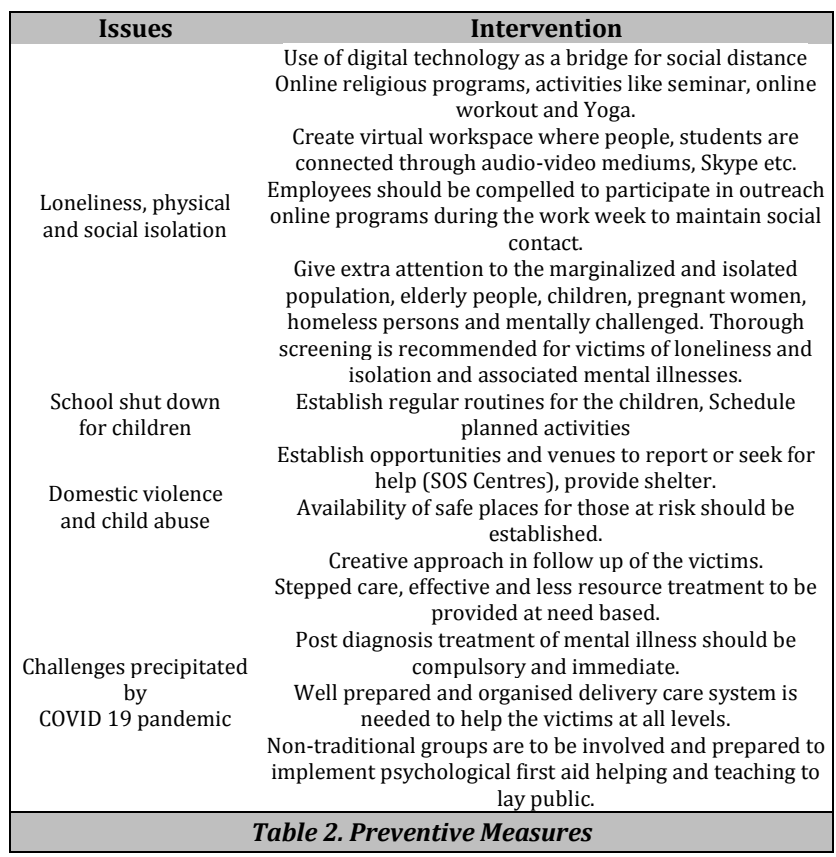

While focusing on curbing the spread of COVID-19, adequate mental health preparedness is needed to address the mental toll due to the pandemic on individuals and communities across the countries.(14) In the context of the COVID-19 pandemic, it is much anticipated that anxiety, depression, substance use, loneliness, and domestic violence will/or already has shown alarming rise; COVID -19 has unveiled number paedophiles with increasing child abuses reported. As a prime concern, psychological first aid and guidance have to be made available with adequate awareness and intervention provided by the mental health care professionals.

Telemedicine, mental health visits, group visits, and delivery of care via technology platforms will be important components of stepped care for both acute crisis management and more routine communication and support. Medicare has already expanded coverage of tele-mental health services and plans to include mental health counseling and virtual visits with psychologists and social workers. Health Care systems, both public and private sector, will need to develop mechanisms for refill and delivery of essential as well as psychiatric medicines. As of now along the prevention-focused approach, population-level and national-level psychological first aid mental health care has to be reinforced with new strategies to emerge from this pandemic.(15)

People who "lose loved ones before their time" are not comforted due to the pandemic crisis. On the contrary, they will be more prone to develop complicated grief that is associated with unexpected death or social isolation or loss of a support system, like what we are experiencing now. The psychological impact is evident on the physical and bio-psychosocial aspects, psycho-neuro-immunity. All forms of psychological support should be routinely implemented in consideration of psychological resilience and also enhancing psycho-neuroimmunity against COVID-19.(16)

\section{Psychological Interventions}

Online platforms providing psychological counseling services for the victims and their families and others affected in the epidemic was established by the PRC. A team of psychological counsellors, nurses, volunteers' psychologists/teachers majoring in psychology and other related fields may not be able to deliver mental health care without professional experiences. The lack of professionals has been a standing problem in the field of psychological intervention. And therefore, many areas may be left out and such problem may precipitate in civil unrest among ignored population.(17)

Focused attention on the consideration of establishment and provision of online mental health services is recommended, its utilization will bring about the necessary changes in the peri and post pandemic scenario. There is still doubtful that online mental health services can improve on mental health service utilization especially in low and middleincome countries (Kauer et al., 2014). Individuals with lower socioeconomic status (SES) might not have as much access to digital technologies for online mental health services. This requires online counseling session by the psychologists which imposes more pressure to the overall quality of online mental health services based on geographical area.(17)

\section{Parenting}

Establish creative opportunity, to build stronger relationships with the younger generation and adolescents at home. Many are collaborating to provide open-access online parenting resources during COVID-19, to be established all over the world according to geographical area. These resources will provide concrete tips to build positive relationships their 
children, to divert and manage bad behaviour, and manage parenting stress. ${ }^{(18)}$

\section{DISCUSSION}

\section{Practical Steps}

No access to official information can foster further anxiety and panic (Johal 2009) but to challenge the pandemic, we have to maintain social distance for self and others safety.(19) Table top exercises are one of the tools designed to simulate the emergence of a public health emergency and address some or all of the phases of emergency management:

- Mitigation.

- Preparedness.

- Response.

- Recovery.

- Table top exercises also require forward thinking and planning in varying scenarios. When a public health emergency occurs, decision makers may be overwhelmed with steps that need their immediate attention. ${ }^{(20)}$

\section{AREAS OF POSSIBLE INTERVENTION BY THE PSYCHIATRISTS}

\section{Educating about the Common Adverse Psychological Consequences}

It is important to inform the public about general measures of countering stress like sleep hygiene, activity scheduling, exercising, social connections, avoiding social media and relaxation techniques. Also, they need to be made aware about the sources and sites available for help.

\section{Encouraging Health-Promoting Behaviours}

People are to be encouraged to take necessary precautions and plan steps ahead in case of such outbreaks. It is also necessary that they know how their workplace / school plan to deal with the onset of epidemic, as it has a reassuring effect on protection for them.

\section{Integrating the Available Healthcare}

Psychiatrists can play an important role in optimizing the standard of care by inter-disciplinary collaboration and education. This will ensure that other medical teams remain sensitized to the mental health perspectives and provide early interventions in the times of distress. Community services can be coordinated to identify health risk and crisis communication.

\section{Facilitate Problem-Solving}

Uncertainty about the future is one of the major factors affecting lives in view of new infections. Self-efficacy measures can be encouraged to prevent hopelessness and social isolation. Early support interventions to be facilitated and family members kept well-informed with comprehensive, updated yet relevant facts will be effective in reducing the fear of uncertainty.

\section{Self-Care of the Health-Care Providers}

Constructive peer-support, supportive therapy and early mental health interventions go a long way to reduce anxiety and to improve quality of care. It is also important for all to stay away from misinformation and on media platforms.(21)

\section{Strengths}

Researchers are very inquisitive about evidences and focus their inquiries regarding COVID - 19 in order to analyse the pandemic at all levels. The findings are very focused and drawn conclusions effectively. Initiatives taken by the government are outstanding steps to minimize the spread. Psychological first aid is very effective method to tone up mental health for both the affected and not affected.

\section{Weakness}

Online mental health services are to be structured as an efficient facility more trials are required perspective of mental health care services. Interventional modules are to be developed to deal with the pandemics. Mental health care pathway in pandemics is to be formulated and established.

\section{CONCLUSIONS}

It is now the time that as mental health caregivers the psychiatrists and professionals, try to integrate with other health-care services keeping mental health at the epicenter. Early identification of distress and timely psychological interventions can not only prevent crisis at times of pandemics but also help in containing its spread and extensive reviews should be carried out.(22)

Financial or Other Competing Interests: None.

\section{REFERENCES}

[1] Zandifar A, Badrfam R. Iranian mental health during the COVID-19 epidemic. Asian J Psychiatr 2020;51:101990.

[2] Jun J, Tucker S, Melnyk BM. Clinician Mental health and well-being during global healthcare crises: evidence learned from prior epidemics for COVID-19 pandemic. Worldviews Evid Based Nurs 2020, DOI: 10.1111/wvn.12439.

[3] Wang C, Pan R, Wan X, et al. Immediate psychological responses and associated factors during the initial stage of the 2019 coronavirus disease (COVID-19) epidemic among the general population in China. Int J Environ Res Public Health 2020;17(5):1729.

[4] Ahmad A, Mueller C, Tsamakis K. Covid-19 pandemic: a public and global mental health opportunity for social transformation? BMJ 2020;369:m1383. 
[5] Venkatesh A, Edirappuli S. Social distancing in covid-19: what are the mental health implications? BMJ 2020;369:m1379.

[6] Li Z, Ge J, Yang M, et al. Vicarious traumatization in the general public, members, and non-members of medical teams aiding in COVID-19 control. Brain Behav Immun 2020;S0889-1591(20)30309-3.

[7] Sani G, Janiri D, Nicola MD, et al. Mental health during and after the COVID-19 emergency in Italy. Psychiatry Clin Neurosci 2020;74(6):372.

[8] Montemurro N. The emotional impact of COVID-19: from medical staff to common people. Brain Behav Immun 2020;87:23-4.

[9] Nussbaumer-Streit B, Mayr V, Dobrescu AI, et al. Quarantine alone or in combination with other public health measures to control COVID-19: a rapid review. Cochrane Database Syst Rev 2020;4(4):CD013574.

[10] Cullen W, Gulati G, Kelly BD. Mental health in the Covid19 pandemic. QJM 2020;113(5):311-2.

[11] Fiorillo A, Gorwood P. The consequences of the COVID-19 pandemic on mental health and implications for clinical practice. Eur Psychiatry 2020;63(1):e32.

[12] Lima CKT, de Medeiros Carvalho PM, de Lima IAAS, et al. The emotional impact of Coronavirus 2019-nCoV (new Coronavirus disease). Psychiatry Res 2020;287:112915.

[13] Li S, Wang Y, Xue J, et al. The Impact of COVID-19 epidemic declaration on psychological consequences: a study on active weibo users. Int J Environ Res Public Health 2020;17(6):2032.

[14] Auerbach J, Miller BF. COVID-19 exposes the cracks in our already fragile mental health system. Am J Public Health 2020;e1-2.

[15] Galea S, Merchant RM, Lurie N. The mental health consequences of COVID-19 and physical distancing: the need for prevention and early intervention. JAMA Intern Med 2020, DOI: 10.1001/jamainternmed.2020.1562.

[16] Kim SW, Su KP. Using psychoneuroimmunity against COVID-19. Brain Behav Immun 2020;87:4-5.

[17] Duan L, Zhu G. Psychological interventions for people affected by the COVID-19 epidemic. Lancet Psychiatry 2020;7(4):300-2.

[18] Yao H, Chen JH, Xu YF. Rethinking online mental health services in China during the COVID-19 epidemic. Asian J Psychiatr 2020;50:102015.

[19] Usher K, Bhullar N, Jackson D. Life in the pandemic: social isolation and mental health. J Clin Nurs 2020, DOI: 10.1111/jocn.15290.

[20] Cluver L, Lachman JM, Sherr L, et al. Parenting in a time of COVID-19. Lancet 2020;395(10231):e64.

[21] Wendelboe AM, Miller A, Drevets D, et al. Tabletop exercise to prepare institutions of higher education for an outbreak of COVID-19. J Emerg Manag 2020;18(2):S1-20.

[22] Banerjee D. The COVID-19 outbreak: crucial role the psychiatrists can play. Asian J Psychiatr 2020;50:102014. 